\title{
Prevalência de lesões em atletas de Taekwondo do estado de Santa Catarina
}

\section{Prevalence of injuries in Taekwondo athletes in the state of Santa Catarina}

\author{
Manuella Manske ${ }^{1}$
}

1 - Centro Universitário Avantis - UNIAVAN, Balneário Camboriú, Santa Catarina, SC, Brasil.

manski.manu@hotmail.com

Palavras-chave:

Traumatismos em Altetas; Aplicações da Epidemiologia; Artes Marciais.

\section{RESUMO}

Introdução: o taekwondo é um esporte de combate competitivo, nas suas quatro vertentes (Kyorugui, Poomsae, Freestyle e Kiopá), com $70 \%$ chutes e $30 \%$ socos com muita explosão na qual ocorrem as disputas entre adversários e oponentes imaginários, sendo o chute como principal movimento de ataque, o contato de alto impacto desse movimento aumenta o risco de lesões. Objetivo: verificar os fatores associados à prevalência de lesões em atletas no taekwondo de Santa Catarina. Método: foi aplicado em questionário via Google Forms incluso ao site da Federação Catarinense de Taekwondo, no qual abordou questões relacionadas ao mecanismo de lesão, segmento mais acometidos, medidas preventivas utilizadas, dominância de membros e período que a lesão ocorreu. Resultados: participaram dessa pesquisa 59 atletas filiados à Federação Catarinense de Taekwondo dentro das categorias juvenil, sub 21, adulto e master de ambos os sexos. Verificou-se que a maior parte dos participantes sofreu algum tipo de lesão proveniente do treino ou competição e casos que impediu o atleta de competir, os locais mais acometidos pelas lesões foram joelhos, pés, tornozelos, posterior de coxa, panturrilhas, mãos, sendo a maior parte das lesões ocorridas em situações de ataques. As dominâncias quanto às lesões que adquire durante o treinamento ou competição de taekwondo e os fatores associados ao tratamento para a prevenção de lesões ocorre maior significância em correlação aos segmentos das lesões apresentadas pelos atletas de categoria juvenil, sub21, adulto e master. Conclusão: a maior prevalência de lesão está na categoria adulto, sendo que a maioria foi impedida de competir. O motivo principal das lesões são gestos de ataque durante treino ou competição.e o localmais acometido é o joelho.

\begin{abstract}
Introduction: taekwondo is a competitive combat sport, in its four aspects (Kyorugui, Poomsae, Freestyle and Kiopá), with 70\% kicks and 30\% punches, and a lot of explosion. In the disputes between opponents and imaginary opponents, kicks are the main attack movement, and the high impact contact of this movement increases the risk of injury. Objective: to verify the factors associated with the prevalence of injuries in athletes in the Santa Catarina Taekwondo Federation. Method: a questionnaire via Google Forms included in the website of the Santa Catarina Taekwondo Federation was applied. It addressed issues related to the injury mechanism, most affected segment, preventive measures used, limb dominance and the period the injury occurred. Results: study participants were 59 athletes affiliated to the Santa Catarina Taekwondo Federation within the youth, under 21, adult and master categories of both sexes. Most participants suffered some type of injury from training or competition and these cases prevented the athletes from competing. The places most affected by the injuries were knees, feet, ankles, posterior thigh, calves, hands, with most injuries occurring in attacks. The predo-

Keywords: $\begin{aligned} & \text { minance of the injuries acquired during training or taekwondo competition and the factors associated } \\ & \text { with the treatment for the prevention of injuries occur with greater significance in correlation to the }\end{aligned}$ Athletes Injuries; Epi- injury segments presented by youth, under-21, adult and master athletes. Conclusion: the highest preMartial Arts. reason for injuries is attack gestures during training or competition. The most affected area is the knee.
\end{abstract} demiology Applications; valence of injury is in the adult category, with the majority being prevented from competing. The main 


\section{INTRODUÇÃO}

O taekwondo é um esporte de combate competitivo, muito popular nas suas quatro vertentes, Kyorugui, Poomsae, Freestyle e kiopá, criado na Coreia do Sul pelo general oficial Choi Hong Hi em $1955,{ }^{1}$ sendo que o mesmo foi reconhecido como um esporte oficial nas Olimpíadas de Sydney. ${ }^{2}$ Esta modalidade é praticada por atletas com diversas idades no mundo todo, englobando um conjunto de características marcantes de golpes com punhos e pés em esforços alternados, os quais apresentam alta intensidade de demanda física durante sua prática. ${ }^{3,1}$

A primeira vertente, o Kyorugui a ênfase durante competição é marcar o maior número de pontos ou nocautear o adversário, para isso é necessária aplicação de chutes no tronco ou cabeça e socos no tronco. Na segunda vertente, o Poomsae o objetivo consiste na execução de uma série de movimentos de ataques e defesas, de acordo com um padrão de movimentos previamente definidos. Já a terceira vertente, o Freestyle é uma apresentação de movimentos básicos com elementos de Kyorugui, Defesa Pessoal, Kiopá e do Poomsae incluídos em uma coreografia. E por último a quarta vertente Kiopá, uma técnica de quebramento com precisão em tábuas (madeira), tijolos e telhas.

Nesse sentido, em combate, a exigência de demandas fisiológicas segundo Campos et al. ${ }^{4}$ são predominantemente aeróbias, contribuindo com cerca de $66 \%$ da demanda energética em combate, enquanto $30 \%$ da demanda energética é anaeróbia, e $4 \%$ láctica. Assim, além do desempenho ser baseado nas condições fisiológicas ele também depende das características técnicas, táticas, e psicológicas do praticante. $^{5}$ Com isso, a intensidade da prática provoca o aumento nas incidências de lesões, devido a diferentes causas como, a falta de preparação física e de orientação adequada dos técnicos e professores. ${ }^{6}$

Neste contexto, observa-se que durante a prática esportiva, é comum a ocorrência de lesões musculoesqueléticas, ${ }^{7}$ que podem afetar o desempenho dos atletas, promovendo diminuição de força, resistência, mobilidade, estabilidade e agilidade durante as suas práticas. ${ }^{8,7}$ A busca por prevenções das lesões esportivas vem sendo a principal preocupação dos atletas de alto rendimento. ${ }^{9}$ A lesão é um acometimento indesejável e desagradável na vida do atleta. ${ }^{10}$ Estudos apontam que as principais lesões que acometem atletas em diferentes práticas esportivas são: distensões, ${ }^{11}$ câimbras ${ }^{12}$ contusão e tendinopatias. ${ }^{13}$

Altarriba-Bartes et al. $^{14}$ indicam que no taekwondo durante a prática o atleta está exposto a riscos permanentes de lesões, evidenciando no estudo de Kazemi, Shearer e Choung ${ }^{15}$ afirmam que $54 \%$ das lesões no taekwondo ocorrem durante o combate, enquanto $36 \%$ das lesões ocorrem durante o treinamento, dessas lesões, cerca de $46,5 \%$ ocorrem em membros inferiores e $18 \%$ em membros superiores devido ao taekwondo ser esporte de combate de percussão com contato direto e ações traumáticas entre dois adversários. ${ }^{15,16}$ Como tal, estudos vêm apresentando o interesse pelas taxas de lesões no esporte, bem como, as intervenções impostas aos atletas com o objetivo de diminuir as taxas de lesões sem diminuir o desempenho do atleta em combate. ${ }^{16-18}$

$\mathrm{O}$ interesse em caracterizar as lesões quanto aos métodos justifica-se porque a prevalência de lesões esportivas pode decorrer por séries de fatores associados que podem apresentar uma infinidade de efeitos adversos ${ }^{19,20}$ como por outro lado organização esportiva, treino técnico, sistema de competição e a falta de estrutura médica podem favorecer fatores de riscos ao atleta envolvido. ${ }^{21}$ Beis et al. ${ }^{22}$ trazem o contexto de que a lesão propriamente dita é a base científica, para que sejam aplicadas a melhor forma de controle de tratamento e também a prevenção. Assim, busca-se nesse estudo trazer uma contribuição para o melhor conhecimento das lesões no taekwondo, permitindo aos profissionais de equipe multidisciplinar aplicar aos seus atletas as medidas preventivas. Deste modo, este estudo tem como objetivo de verificar a prevalência de lesões dos atletas filiados à Federação Catarinense de Taekwondo (FCTKD) e investigar os segmentos das lesões que ocorrem durante o treinamento ou competição apresentados pelos atletas de alto rendimento da modalidade de taekwondo, por meio do mecanismo de ocorrência, membro de dominância da modalidade e verificar as formas de tratamento para a prevenção como propósito do desempenho esportivo.

\section{MÉTODO}

\section{Caracterização do estudo}

Trata-se um estudo do tipo descritivo de caráter transversal para avaliação da prevalência de lesões em atletas de taekwondo presentes em todos os municípios de Santa Catarina e filiados à Federação 
Catarinense de Taekwondo (FCTKD). O estudo foi aprovado pelo Comitê de Ética em Pesquisa do Centro Universitário Avantis (UNIAVAN) sob o CAAE n ${ }^{\circ}$ 12974719.1.0000.5592.

\section{Procedimentos de coleta de dados}

A FCTKD foi esclarecida sobre a natureza da pesquisa, seus objetivos, procedimento quanto ao aceite em participar do estudo disponibilizando para os atletas filiados e competidores. Após esclarecimentos, foi aberto o questionário validado junto com o termo de consentimento livre e esclarecido via online no Google forms e mencionado ao site da FCTKD para que os atletas pudessem obter o acesso, garantindo-se o anonimato e a liberdade de ausência na pesquisa, sendo que foi garantido o direito de desistir de sua participação a qualquer momento e os dados foram utilizados para a determinação dos critérios de inclusão e exclusão.

\section{Critérios de inclusão e exclusão}

Os critérios de inclusão foram:

- Estar regularmente filiados a FCTKD;

- Categoria Juvenil, Sub 21, Adulto e Master com idade de 18 a 60 anos;

- Competidores de graduação acima de $2^{\circ}$ Gub Vermelha.

Os critérios de exclusão foram:

- Menor de 18 Anos;

- Competidores de graduação abaixo de $3^{\circ}$ gub - Azul ponta Vermelha.

\section{Participantes do estudo}

Participaram do de presente estudo 59 atletas de taekwondo sendo 39 homens e 20 mulheres de diversas academias situadas em 19 municípios de Santa Catarina. Ressalta-se que responderam ao questionário 63 atletas, no entanto, 4 desses pertenciam a categoria cadete (idade menor que 18 anos) assim esses não foram inclusos na pesquisa.

\section{Análises de dados}

Após a coleta de dados, os mesmos foram organizados e tabulados em uma planilha do Excel (Office Microsoft, versão 2010). Para a análise dos dados foi utilizado o software estatístico JASP 8.0. Para a descrição dos participantes da pesquisa, e verificação da prevalência dos principais locais de lesão em atletas de taekwondo adotou-se estatística descritiva por meio de medida de tendência central (média) e dispersão (desvio padrão), bem como, por meio de distribuição (frequência relativa e frequência absoluta). Para verificar possíveis fatores associados à ocorrência ou não de lesão utilizou-se o teste do QuiQuadrado ou Exato de Fischer quando necessário. O valor de significância do teste foi definido em $5 \%$.

\section{RESULTADOS}

Dos 59 atletas participantes na pesquisa foram divididos entre categorias juvenil, adulto, sub 21 e master totalizando $66,1 \%$ homens e $33,9 \%$ mulheres

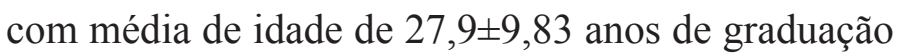
vermelha $2^{\circ} \mathrm{Gub}$ à preta $6^{\circ}$ Dan, competidores com frequência semanal de treino 4,12 $\pm 1,61$ dias por semana e 2,12 $\pm 1,33$ horas diárias de treinamentos

Tabela 1 - Caracterização dos participantes

\begin{tabular}{|c|c|c|c|}
\hline & & & n $(\%)$ \\
\hline \multirow{2}{*}{ Sexo } & & Homens & $39(66,1)$ \\
\hline & & Mulheres & $20(33,9)$ \\
\hline \multirow{5}{*}{ Categoria } & & Juvenil & $9(15,2)$ \\
\hline & & Sub 21 & $6(10,1)$ \\
\hline & & Adulto & $16(27,2)$ \\
\hline & & Master & $28(47,5)$ \\
\hline & Geral X (dp) & Homens X (dp) & Mulheres X (dp) \\
\hline Idade & $27,9(9,83)$ & $31,46(9,18)$ & $21,22(7,30)$ \\
\hline Frequência semanal de treino & $4,12(1,61)$ & $3,97(1,66)$ & $4,42(1,50)$ \\
\hline Horas por dia de treino & $2,12(1,33)$ & $2,14(1,40)$ & $2,08(1,25)$ \\
\hline
\end{tabular}

$\mathrm{X}=$ Média, $\mathrm{dp}=$ desvio padrão 
Quando aos fatores de dominância de Taekwondo, os achados da figura 1 relata o lado para preferência ao chutar ou socar (ataque/defesa) os membros inferiores e superiores.

os atletas filiados a Federação Catarinense de

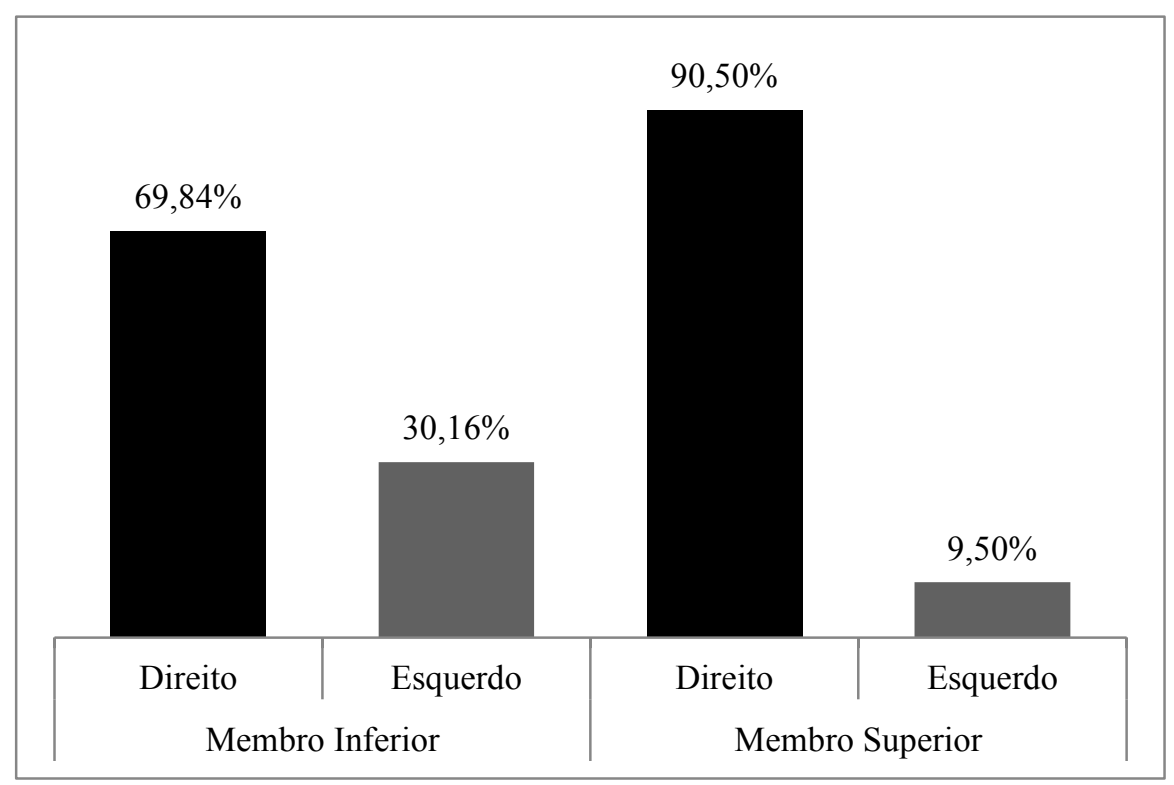

Figura 1 - Membros Superiores e Inferiores dominantes dos Atletas filiados à Federação Catarinense de Taekwondo

Entre categorias verifica-se na tabela 2, a prevalência de lesões contemplam a categoria adulta, tiveram alguma lesão, seguido atrás categoria sub 21 e por ultimo a categoria master $78,6 \%$ sofreram lesões quase semelhante à categoria juvenil por poucas diferenças de porcentagem em concorrência obtém $77,8 \%$.

Tabela 2 - Prevalência de Lesão por Categoria.

\begin{tabular}{|c|c|c|}
\hline & Lesão proveniente de treino ou competição & n $(\%)$ \\
\hline \multirow{2}{*}{ Juvenil } & Sim & $7(77,8)$ \\
\hline & Não & $2(22,2)$ \\
\hline \multirow{2}{*}{ Sub 21} & Sim & $5(83,3)$ \\
\hline & Não & $1(16,7)$ \\
\hline \multirow{2}{*}{ Adulto } & Sim & $16(100,0)$ \\
\hline & Não & - \\
\hline \multirow{2}{*}{ Master } & Sim & $22(78,6)$ \\
\hline & Não & $6(21,4)$ \\
\hline
\end{tabular}

assim como outras características estão descritas conforme tabela 1 .

Os locais de acometimentos das lesões pelos atletas filiados a Federação Catarinense de Taekwondo durante treino ou competições, descritas na tabela 3 , além dessas estimativas observa-se $84,7 \%$ já sofreram algum tipo de lesão proveniente do treino ou competição, sendo que em $62,7 \%$ dos casos a lesão impediu o atleta de competir, resultam os segmentos corporais de maior frequência em $1^{\circ}$ lugar Joelhos 39 $(66,1 \%), 2^{\circ}$ lugar os pés $32(54,2 \%), 3^{\circ}$ lugar empate de tornozelos e posterior de coxa $24(40,6 \%), 4^{\circ}$ lugar panturrilhas $20(33,9 \%), 5^{\circ}$ mãos $18(30,5 \%)$ e por último $6^{\circ}$ lugar ombros $(23,7 \%)$. 
Tabela 3 - Frequência de lesão dos atletas de taekwondo catarinense e os principais pontos de lesão.

\begin{tabular}{|c|c|c|}
\hline & & n $(\%)$ \\
\hline \multirow{2}{*}{ Lesão proveniente de treino ou competição } & Sim & $50(84,7)$ \\
\hline & Não & $9(15,3)$ \\
\hline \multirow{2}{*}{ A lesão impediu o atleta de competir } & Sim & $37(62,7)$ \\
\hline & Não & $22(37,3)$ \\
\hline \multirow{2}{*}{ Lesão nos ombros } & $\operatorname{Sim}$ & $14(23,7)$ \\
\hline & Não & $45(76,3)$ \\
\hline \multirow{2}{*}{ Lesão nos braços } & Sim & $10(16,9)$ \\
\hline & Não & $49(83,1)$ \\
\hline \multirow{2}{*}{ Lesão nos antebraços } & Sim & $7(11,8)$ \\
\hline & Não & $52(88,2)$ \\
\hline \multirow{2}{*}{ Lesão nos cotovelos } & Sim & $7(11,8)$ \\
\hline & Não & $52(88,2)$ \\
\hline \multirow{2}{*}{ Lesão nos punhos } & Sim & $10(16,9)$ \\
\hline & Não & $49(83,1)$ \\
\hline \multirow{2}{*}{ Lesão nas mãos } & Sim & $18(30,5)$ \\
\hline & Não & $41(69,4)$ \\
\hline \multirow{2}{*}{ Lesão no tórax } & Sim & $9(15,2)$ \\
\hline & Não & $50(84,8)$ \\
\hline \multirow{2}{*}{ Lesão abdômen } & Sim & $7(11,8)$ \\
\hline & Não & $52(88,2)$ \\
\hline \multirow{2}{*}{ Região cervical } & Sim & $7(11,8)$ \\
\hline & Não & $52(88,2)$ \\
\hline \multirow{2}{*}{ Região dorsal } & Sim & $8(13,5)$ \\
\hline & Não & $51(86,5)$ \\
\hline \multirow{2}{*}{ Região Lombar } & Sim & $9(15,2)$ \\
\hline & Não & $50(84,8)$ \\
\hline \multirow{2}{*}{ Quadril } & Sim & $9(15,2)$ \\
\hline & Não & $50(84,8)$ \\
\hline \multirow{2}{*}{ Anterior de coxa } & Sim & $11(18,6)$ \\
\hline & Não & $48(81,4)$ \\
\hline \multirow{2}{*}{ Posterior de coxa } & Sim & $24(40,6)$ \\
\hline & Não & $35(59,4)$ \\
\hline \multirow{2}{*}{ Joelho } & Sim & $39(66,1)$ \\
\hline & Não & $20(33,9)$ \\
\hline \multirow{2}{*}{ Panturrilha } & Sim & $20(33,9)$ \\
\hline & Não & $39(66,1)$ \\
\hline \multirow{2}{*}{ Tornozelo } & Sim & $24(40,6)$ \\
\hline & Não & $35(59,4)$ \\
\hline \multirow{2}{*}{ Pés } & Sim & $32(54,2)$ \\
\hline & Não & $27(45,8)$ \\
\hline
\end{tabular}

Durante o treinamento ou competição, o na figura 2, ocorrendo com maior frequência no gesto mecanismo de ocorrência das lesões dos atletas filiados de ataque. a Federação Catarinense de Taekwondo, verifica-se 


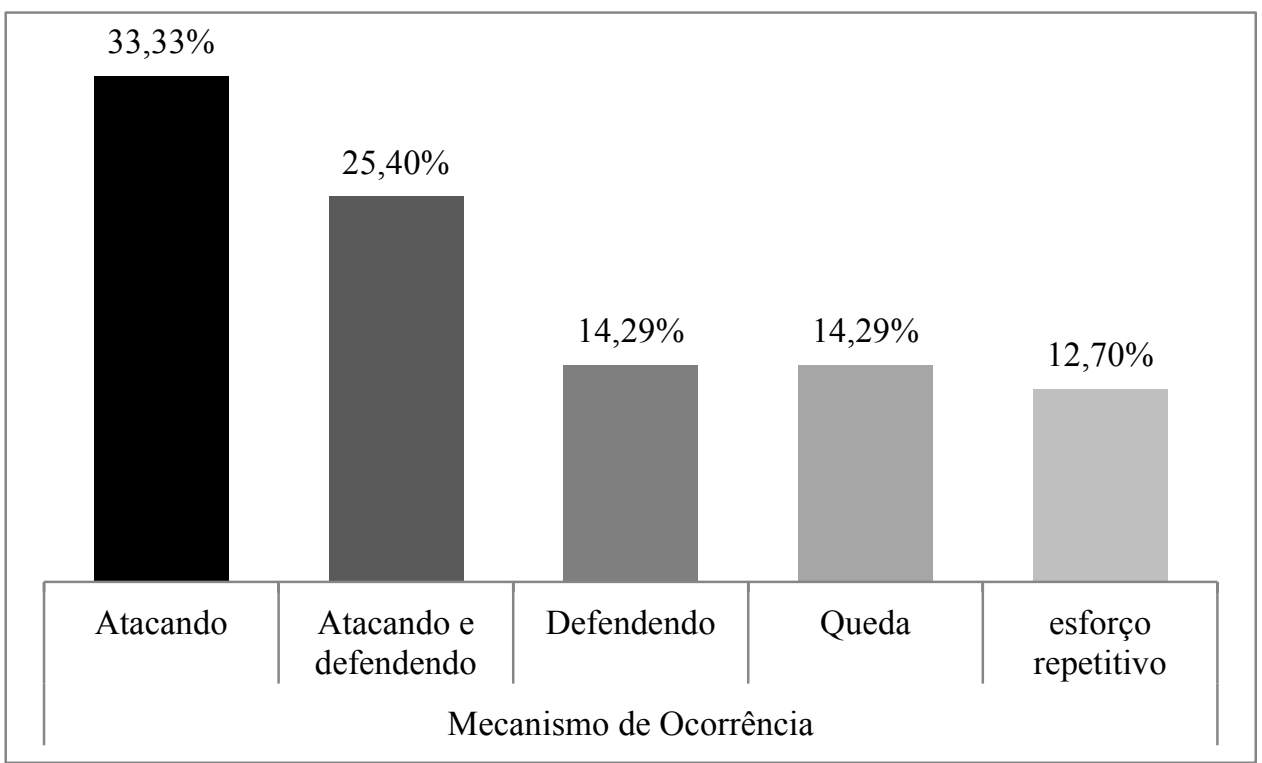

Figura 2 - Mecanismos de ocorrência de lesões dos atletas filiados a Federação Catarinense de Taekwondo

Na tabela 4 são apresentados os fatores de treinamento/acompanhamento que são associados à lesão no taekwondo, sendo que o trabalho muscular compensatório foi à única variável que se apresentou significativamente associada à presença ou não de lesão, ou seja, os atletas que realizam trabalhos musculares compensatórios parecem ter menor risco de sofrer lesões.

Tabela 4 - Fatores associados à lesão no taekwondo.

\begin{tabular}{|c|c|c|c|c|c|}
\hline & & \multicolumn{2}{|c|}{$\begin{array}{c}\text { Sofreu lesão conforme treino / } \\
\text { acompanhamento }\end{array}$} & \multirow[b]{2}{*}{$\mathrm{X}^{2}$} & \multirow[b]{2}{*}{ p-valor } \\
\hline & & Sim n (\%) & Não n (\%) & & \\
\hline \multirow{2}{*}{ Treinamento Físico (musculação) } & Sim & $32(86,4)$ & $5(13,6)$ & \multirow{2}{*}{0,30} & \multirow{2}{*}{0,58} \\
\hline & Não & $18(81,8)$ & $4(18,2)$ & & \\
\hline \multirow{2}{*}{ Treinamento Aeróbio } & Sim & $32(82,0)$ & $7(18,0)$ & \multirow{2}{*}{0,34} & \multirow{2}{*}{0,55} \\
\hline & Não & $18(90,0)$ & $2(10,0)$ & & \\
\hline \multirow{2}{*}{ Treinamento de Flexibilidade } & $\operatorname{Sim}$ & $38(82,6)$ & $8(17,4)$ & \multirow{2}{*}{0,23} & \multirow{2}{*}{0,63} \\
\hline & Não & $12(92,3)$ & $1(7,7)$ & & \\
\hline \multirow{2}{*}{ Acompanhamento Médico } & $\operatorname{Sim}$ & $5(83,3)$ & $1(16,7)$ & \multirow{2}{*}{0,36} & \multirow{2}{*}{0,54} \\
\hline & Não & $45(84,9)$ & $8(15,1)$ & & \\
\hline \multirow{2}{*}{ Acompanhamento com Fisioterapeuta } & Sim & $17(89,4)$ & $2(10,6)$ & \multirow{2}{*}{0,22} & \multirow{2}{*}{0,63} \\
\hline & Não & $33(82,5)$ & $7(17,5)$ & & \\
\hline \multirow{2}{*}{ Acompanhamento Preparador Físico } & Sim & $22(84,6)$ & $4(15,4)$ & \multirow{2}{*}{0,06} & \multirow{2}{*}{0,59} \\
\hline & Não & $28(84,9)$ & $5(15,1)$ & & \\
\hline \multirow{2}{*}{ Acompanhamento Prof. Ed. Física } & Sim & $26(89,6)$ & $6(10,4)$ & \multirow{2}{*}{0,50} & \multirow{2}{*}{0,47} \\
\hline & Não & $24(88,8)$ & $3(11,2)$ & & \\
\hline \multirow{2}{*}{ Acompanhamento Treinador } & Sim & $40(88,8)$ & $5(11,2)$ & \multirow{2}{*}{2,83} & \multirow{2}{*}{0,10} \\
\hline & Não & $10(71,4)$ & $4(28,5)$ & & \\
\hline \multirow{2}{*}{ Trabalho Muscular Compensatório } & Sim & $15(71,4)$ & $6(28,6)$ & \multirow{2}{*}{4,23} & \multirow{2}{*}{$0,04 *$} \\
\hline & Não & $35(92,1)$ & $3(7,9)$ & & \\
\hline \multirow{2}{*}{ Fisioterapia Preventiva } & Sim & $15(88,2)$ & $2(11,8)$ & \multirow{2}{*}{0,05} & \multirow{2}{*}{0,56} \\
\hline & Não & $35(83,4)$ & $7(16,6)$ & & \\
\hline
\end{tabular}

$\mathrm{X}^{2}=$ teste do qui-quadrado. ${ }^{*} \mathrm{p}$-valor $<0,05$. 


\section{DISCUSSÃO}

Este estudo objetivou verificar a prevalência de lesões entre categoria Juvenil, Sub 21, Adulto e Master dos atletas graduados e competidores filiados à Federação Catarinense de Taekwondo do Estado de Santa Catarina. Verificou-se que os atletas ao chutar ou socar (ataque/defesas) dominam o lado direito como ponto forte de preferência e os que apresentaram a maior prevalência de lesão foram à categoria adulta, observou-se maioria dos atletas sofreu lesões que os impediram de competir, ainda foi evidenciado que o joelho é o ponto alvo da maioria das lesões da modalidade de Taekwondo, sendo que o motivo principal das lesões são gestos de ataque durante treino ou competição. Observou-se ainda uma associação entre a realização de trabalho muscular compensatório e presença ou não de lesão.

Foi possível, identificar-se um alto percentual de atletas homens comparado a atletas mulheres, essa tendência já vem dos primórdios das artes marciais, uma vez que, nessas modalidades com características da defesa pessoal que contam com proteções e formas de combate de alto risco e muito contato físico ${ }^{23,24}$ existindo uma predominância de praticantes homens, ${ }^{25}$ que permite adaptar a carreira esportiva em especialização ao alto rendimento em uma ou as duas: poomsae e kyorugui.

Devido à utilização prioritária de golpes no contexto socar e chutar, os atletas predominam o lado direito em ação de ataques e defesas, com maior capacidade de explosão e flexibilidade, além das técnicas exigindo agilidade, velocidade e resistência ao corpo e cabeça com a demanda de treinamentos, apoia segundo estudos Barbieri et al. ${ }^{26,27}$ a maioria é destro como componente forte dos membros para a realização dos movimentos de chute como dominante, e o membro não dominante para o suporte e estabilização do corpo.

Nota-se que a categoria adulta com a maior prevalência de lesões, pois nessa categoria grande parte dos atletas não realizam outros tipos de exercícios a não ser a modalidade em específica taekwondo, intensificou-se a sobrecarga física e psicológica que traz no sentido como requisitos uma combinação indeterminada de ações e interações, reação instantânea as dificuldades pelo opositor e as adversidades da luta. Smaniotto ${ }^{28}$ verificou que muitos atletas se lesionam em academias durante os treinos e chegam aos campeonatos lesionados, já Onofre e Navarro ${ }^{29}$ afirmam que em alguns casos o atleta lesiona-se durante o combate.

Nesse sentido no presente estudo se observa que muitos atletas que participam nessa pesquisa sofreram algum tipo de lesão proveniente do treino ou competição, sendo ainda que na maior parte dos casos a lesão impediu o atleta de competir. Para os combates os atletas utilizam proteções nas pernas, protegendo tíbia e fíbula, no antebraço, protegendo rádio e ulna, proteção nos pés, capacete de proteção na cabeça, luva de proteção nas mãos, protetor bucal, protetor genital e protetor de tórax, ${ }^{30}$ porém, em certos momentos, este equipamento é insuficiente para evitar o aparecimento de lesões. ${ }^{12}$

Ainda se observa no presente estudo que os locais com maior frequência de lesão nos atletas de taekwondo foram joelho, pés, tornozelos e posterior de coxa, panturrilhas, mãos e ombros.Verificou-se também que os mecanismos de ocorrência de lesões são predominantemente em ações de ataques, no momento do treino ou competição.

Esses resultados corroboram com estudos $^{28,31-33,14}$ que evidenciaram que os principais traumas acometidos em atletas de Taekwondo eram em: pé, tornozelo e joelho. O joelho é uma articulação com constante risco de lesão, pois é uma zona anatômica muito afetada durante a prática do taekwondo, tanto em período de treinamento como em competições, ${ }^{35-37}$ uma vez que, os atletas possuem grande solicitação das estruturas articulares e tendíneo-musculares, que permitem grandes amplitudes de movimento, ${ }^{38,39}$ exigindo um trabalho biomecânico podendo ser lesionado de várias formas por ser muito vulnerável ao trauma direto (pancadas) ou indireto (entorse) atribuída a grande magnitude das força de impactos presentes no membro inferior.

Por várias vezes em uma ação de defesa, os joelhos e cotovelos são utilizados para bloquear os golpes, no taekwondo cerca de $98 \%$ das pontuações são por meio de chutes durante kyorugui. Quanto às lesões nos pés e tornozelos, justifica-se a grande ocorrência pelas ações específicas da modalidade..$^{40}$ Listam como exemplo os chutes mais altos, na região da cabeça: An Tchagui, Bakat Tchagui, Bandal Tchagui, Dolio Tchagui, Furyo Tchagui, Mondolio Tchagui, Neryo Tchagui, Tigô Tchagui e Timio Mondolio Ruryo Tchagui são bem procurados durante Kyorugui, por serem golpes que mais 
pontuam, estes chutes solicitam maior flexibilidade e maior força muscular do atleta, por necessitar de mais força e flexibilidade, pode conduzir a um maior índice de traumas como "distensões e contraturas decorrentes dos esforços destas técnicas, tendo assim grande importância da flexibilidade dentro desta arte marcial" ${ }^{11}$ e no caso de Poomsae e Freestyle reverte ao oponente imaginário quando executa uma gama de golpes deferidos, e serve também aos movimentos ao realizar o Kiopá aplicado em tábuas de forma incorreta aos movimentos bruscos.

Já as ocorrências de lesões nas mãos podem estar relacionadas ao gesto de defesa, quando podem ocorrer traumas diretos como movimento de defesa do chute adversário em casos de kyorugui. ${ }^{15} \mathrm{Ou}$ ainda, ao executar a técnica kiopá com socos aplicados nas tábuas e principalmente em telhas e tijolos gerando assim lesões traumáticas. ${ }^{42}$

Nesse sentido sugere-se a necessidade de um trabalho compensatório na prevenção de lesões atuando no sentido de minimizar os efeitos sobrecarga repetitivas do treinamento para a execução perfeita dos elementos de chutes como socos. Uma vez que esses movimentos irão compensar a tensão gerada pelo uso excessivo assim como a fadiga muscular, reequilibrando-os, sendo pouco valorizada pela maioria dos treinadores e atletas por apresentar debilidade na quantidade de treino semanal, além de poucas atividades destinadas a prevenção e melhora do condicionamento físico, pelo fato que houve maiores diferenças dos membros superior e inferior de dominância fortalecedora dos atletas que são de preferência do lado direito, visto que ser mais fácil para esse grupo executar técnicas com maior frequência.

Assim a importância dos exercícios compensatórios específicos como foi evidenciado no presente estudo, são de extrema importância, já que a compensação aos esforços repetitivos e desequilíbrios posturais como fortalecimento muscular no lado menos solicitado, principalmente membros inferiores, assim como aprimoramento técnico dos gestos esportivos com objetivo de melhorar a saúde e compensar os efeitos negativos do movimento estão associados a uma menor ocorrência de lesões. Tamborindey et al. ${ }^{43}$ sugerem a efetivação do trabalho compensatório para prevenir lesões e desvios posturais em atletas de taekwondo.

Deste modo, adicionar a inclusão desses exercícios compensatórios pode ser uma estratégia para os treinadores e profissional da área para prevenir e retardar a instalação de lesões assim como amenizar as que já são instaladas. A percepção do próprio atleta sobre sua lesão pode auxiliar em processos futuros de prevenção. ${ }^{44}$

\section{CONCLUSÃO}

Conclui-se a partir do presente estudo que a maior prevalência de lesão está na categoria adulto, ainda se verificou que a maioria ficou impedida de competir por conta da lesão, ainda foi evidenciado que o joelho é o ponto alvo da maioria das lesões da modalidade de taekwondo, sendo que o motivo principal das lesões são gestos de ataque durante treino ou competição. Por fim uma associação entre a realização de trabalho muscular compensatório e presença ou não de lesão foi encontrada. Assim o fortalecimento muscular compensatório nos atletas de taekwondo é de extrema importância para evitar ocorrências de lesões, além da gama de exercícios preventivos que os atletas conhecem como: alongamento e relaxamento que já fazem no início e final de treinos e competições, treinamento aeróbio e alguns casos de correção postural até treinamento proprioceptivo nas academias, entre outros, sob a orientação, conduzidos por profissionais integrantes de equipe multidisciplinar. Sugere-se para treinadores e preparadores físicos que deem preferência ao membro não dominante dos atletas nos trabalhos compensatórios. Ainda se recomenda para estudos futuros que estendam a avaliação para a categoria infantil e cadete e para um numero maior de atletas.

\section{Agradecimentos}

Quero agradecer ao meu Professor de taekwondo Felipe Cunha Santos, inicialmente ele abriu a ideia do meu projeto, que me ajudou no decorrer desse artigo de TCC, ao meu Fisioterapeuta Rodrigo Bergenthal que me apoiou nesse projeto e me tratou de uma lesão de Joelho LCA devido ao treino de taekwondo, e por eles dou mérito e minha gratidão por me ajudar nesse momento do ano. A Federação Catarinense por incluir esse projeto aos atletas para que contribuísse um levantamento histórico com bases científicas e que pudesse demostrar a todos, a minha gratidão ao Mestre e Presidente Allan Fábio Siqueira da Federação Catarinense de Taekwondo. E por fim a minha família, entender que um trabalho e preciso 
de muita força e superação a cada dia, obrigada por me aguentar meus neurônios e o meu tempo nesse processo de trabalho.

\section{REFERÊNCIAS}

1. Dias HM, Ramallo BT, Zanetti MC, Figueira Junior AJ, Brandão MRF, Marin DP, Polito LFT. Systematic review of means and methods of practical influence on taekwondo training. J Phys Educ 2018, 29(1):1-14. doi: https://doi. org/10.4025/jphyseduc.v29i1.2916

2. Kim HB, Johnson JA, Lee EJ, Ha P. An investigation into the history of the taekwondo uniform since the Korean Peninsula's liberation from Japan. Int J Hist Sport 2016; 33(9):963-77. doi: https://doi.org/10.1080/09523367.2016.1233865

3. Costa DO, Oliveira LS, Sena EA, Lima FF, Silva AS. Precompetition physical, physiological and psychosocial states of taekwondo athletes. J Phys Educ 2018;29(1):1-11. doi: https:// doi.org/10.4025/jphyseduc.v29i1.2913

4. Campos FAD, Bertuzzi R, Dourado AC, Santos VGF, Franchini E. Energy demands in taekwondo athletes during combat simulation. Eur J Appl Physiol 2012;112(4):1221-8. doi: https://doi.org/10.1007/s00421-011-2071-4

5. Bridge CA, Santos JFS, Chaabène H, Pieter W, Franchini E. Physical and physiological profiles of taekwondo athletes. Sports Med 2014;44(6):713-33. https://doi.org/10.1007/s40279014-0159-9

6. Cohen M, Abdala RJ. Lesões no esporte: diagnóstico, prevenção e tratamento. Rio de Janeiro: Revinter. 2003. 1152 p. 7. Shirabe NA, Silva RA, Oliveira MR, Nowotny AH, Sturion LA, Gil AWO, Andraus RAC, Carvalho APF. Atletas de taekwondo têm melhor controle postural do que atletas de handebol e futebol americano. Rev Bras Med Esporte 2017;23(6):473-6. doi: http:// dx.doi.org/10.1590/1517-869220172306170049

8. Arnold BL, Motte SDL, Linens S, Ross SE. Ankle instability is associated with balance impairments: a meta-analysis. Med Sci Sports Exerc 2009;41(5):1048-62. https://doi.org/10.1249/ MSS.0b013e318192d044

9. Silfies SP, Ebaugh D, Pontillo M, Butowicz CM. Critical review of the impact of core stability on upper extremity athletic injury and performance. Braz J Phys Ther 2015;19(5):360-8. doi: https://doi.org/10.1590/bjpt-rbf.2014.0108

10. Xavier AA, Lopes AMC. Lesões Musculoesqueléticas em Praticantes de Crossfit. Revista Interdisciplinar Ciências Médicas 2017;1(11):11-27.

11. Arena SS, Carazzato JG. A relação entre o acompanhamento médico e a incidência de lesões esportivas em atletas jovens de São Paulo. Rev Bras Med Esporte 2007;13(4):217-21. doi: https://doi.org/10.1590/S1517-86922007000400001

12. Lystad RP, Pollard H, Graham PL. Epidemiology of injuries in competition taekwondo: A meta-analysis of observational studies. J Sci Med Sport 2009;12(6):614-21. doi: https://doi. org/10.1016/j.jsams.2008.09.013

13. Kordi R, Maffulli N, Wroble RR, Wallace WA, Pieter W. Taekwondo. In: Kordi R, Maffulli N, Wroble RR, Wallace WA, orgs. Combat sports medicine. Londres: Springer; 2009. 263-86. 14. Altarriba-Bartes A, Drobnic F, Til L, Malliaropoulos N, Montoro JB, Irurtia A. Epidemiology of injuries in elite taekwondo athletes: Two Olympic periods cross-sectional retrospective study. BMJ Open 2014;4(2):e004605. doi: http:// dx.doi.org/10.1136/bmjopen-2013-004605

15. Kazemi M, Sherer H, Choung YS. Pre-competition habits and injuries in Taekwondo athletes. BMC Musculoskeletal Disorders 2005;6(1):26.

16. Pieter W, Fife GP, O'Sullivan DM. Competition injuries in taekwondo : a literature review and suggestions for prevention and surveillance. Br J Sports Med 2012;46(7):485-91.

17. Feehan M, Waller AE. Precompetition injury and subsequent tournament performance in full-contact taekwondo. Br J Sports Med 1995;29(4):258-62. https://doi.org/10.1136/bjsm.29.4.258 18. Zemper ED, Pieter W. Injury rates during the 1988 US Olympic Team Trials for taekwondo. Br J Sports Med 1989;23(3):161-4. doi: https://doi.org/10.1136/bjsm.23.3.161

19. Joseph C, Finch CF. Sports Injuries. The International Encyclopedia of Public Health 2016;79-86.

20. Keogh JWL, Winwood PW. The epidemiology of injuries across the weight-training sports. Sports Med 2011;47(3):479501. doi: https://doi.org/10.1007/s40279-016-0575-0

21. Oliveira E, Oliveira RRC, Silva KAF. Prevalência e incidência de lesões em atletas participantes do campeonato open de Jiu-Jitsu da cidade de Catalão-go realizado em agosto de 2010. Saúde CESUC, 2010.

22. Beis K, Pieter W, Abatzides G. Taekwondo techniques and competition characteristics involved in time-loss injuries. J Sports Sci Med 2007;6(CSSI-2):45-51.

23. Goulart, Fabio. Tae kwon do: Guia Prático de defesa pessoal. Editora Escala. 2006. 34

24. Goulart, F, Campos T. Taekwondo: O caminho dos pés e das mãos. Ed. On-line. 2007.

25. Kazemi M, Pieter W. Injuries at a Canadian National Taekwondo Championships: A Prospective Study. BMC Muskuloskelet Disorders 2004;5(1):22. Doi: https://doi. org/10.1186/1471-2474-5-22

26. Barbieri FA, Lima Junior RS, Gobbi LTB. Aspectos da corrida de aproximação entre o chute realizado com o membro dominante e não dominante. Revista Motricidade 2006;2(2):8090.

27. Barbieri FA, Santiago PRP, Gobbi LTB, Cunha AS. Diferenças entre o chute realizado com o membro dominante e não-dominante no futsal: Variabilidade, velocidade linear das articulações, velocidade da bola e desempenho. Revista Brasileira de Ciências do Esporte 2008;29(2):129-46.

28. Smaniotto LF. Incidência de Lesões no Taekwondo. XI Brazil Open Taekwondo, p. 12, 2006.

29. Onofre CP, Navarro F. O efeito de um programa de exercícios físicos na sala de musculação para diminuição da dor durante o treinamento e luta de taekwondo: um estudo de caso de lesão de joelho. Revista Brasileira de Prescrição e Fisiologia do Exercício 2008;2(10):420-8.

30. Goulart Fabio. Guia Prático de Defesa Pessoal. São Paulo: Escala, 2005.

31. Costa RSD, Campos RG, Freitas DG. Prevalência de queixas de dor musculoesqueléticas em atletas de taekwondo. Revista Funcional 2009;2(2):33-42.

32. Cavalheiro JENS, Toigo AM. Influência do tipo de piso em lesões nos membros inferiores de praticantes de tae kwon do chute bandal tchagui. EFDeportes.com, Revista Digital 2009;14(139): s/p. 
33. Neto JFO, Campos LAS. Análise da ocorrência de contusões traumáticas no taekwondo no contexto da competição esportiva. Coleção pesquisa em educação física 2013;12(4):49-56.

34. Oliveira Neto JF, Campos LAS. Análise da ocorrência de contusões traumáticas no taekwondo no contexto da competição esportiva. Coleção Pesquisa em Educação Física 2013;12(4):4956.

35. Kazemi M, Chudolinski A, Turgeon M, Simon A, Ho E, Coombe L. Nine year longitudinal retrospective study of Taekwondo injuries. J Can Chirop Assoc 2009;53(4):272-81.

36. JungeA, Engebretsen L, Mountjoy ML, Renström PAJ, Dvorak J. Sports injuries during the summer Olympic games 2008. The American journal of sports medicine 2009;37(11):2165-72. Doi: https://doi.org/10.1177/0363546509339357

37. Ziaee V, Rahmani SH, Rostami M. Injury rates in iranian taekwondo athletes; a prospective study. Asian J Sports Med 2010;1(1):23-8. doi: https://doi.org/10.5812/asjsm.34877

38. Blankevoort L, Huiskes R, De Lange A. Recruitment of knee joint ligaments.1991.

39. Friederich NF, Muller W, O'brien WR. Clinical application of biomechanic and functional anatomical findings of the knee joint. Der Orthopade 1992; 21(1):41-50.

40. Leichtweis M, Antunes B, Xavier B, Del Vecchio F. Efeitos de diferentes protocolos no tempo para executar o chute no taekwondo. Arquivos de Ciências do Esporte. 2013;1(1):37-45.

41. Farias GM. Exercícios proprioceptivos e pliométricos como prevenção de lesão para atletas de taekwondo: Uma proposta de tratamento fisioterapêutico. [Monografia] Trabalho de Conclusão de Curso de Pós-Graduação em Fisioterapia Traumato Ortopédica e Esportiva da Universidade do Extremo Sul Catarinense - UNESC: Criciúma; 2010.

42. Oliveira F C. L. Epidemiologia das lesões nos atletas de taekwondo. [Monografia] 28f. Trabalho de Conclusão de Curso do Graduação em Fisioterapia da Universidade Presidente Antônio Carlos: Juiz de Fora; 2004.

43. Tamborindeguy AC, Tirloni AS, Reis DC, Freitas CDLR, Moro ARP, Santos SG. Incidência de lesões e desvios posturais em atletas de taekwondo. Rev Bras Ciênc Esporte 2011;33(4):97590. doi: https://doi.org/10.1590/S0101-32892011000400012

44. Pastre CM, Carvalho G, Monteiro H, Netto Junior J, Padovani G. Lesões desportivas na elite do atletismo brasileiro: estudo a partir de morbidade referida. Rev Bras Med Esporte 2005;11(1):43-7. doi: https://doi.org/10.1590/S151786922005000100005

Recebido em: 18/12/2019

Aceito em:30/09/2020

Como citar: MANSKE, Manuella. Prevalência de lesões em atletas de taekwondo de Santa Catarina. Revista Interdisciplinar de Promoção da Saúde, Santa Cruz do Sul, v. 2, n. 4, out. 2019. ISSN 2595-3664. Disponível em: <https://online.unisc.br/seer/ index.php/ripsunisc/article/view/14625>. Acesso em: 01 out. 2019. doi:https://doi.org/10.17058/rips.v2i4.14625 